\title{
RESULTADOS PRELIMINARES DO EMPREGO DO ACTH NAS DEGENERAÇÕES COMBINADAS DA MEDULA
}

\author{
Horacio Martins Canlitas * \\ José Antonio Levy*
}

Inicialmente, desejamos frisar que, sob o título dêste trabalho, deve ser entendido que nos referimos aos resultados imediatos do emprêgo do hormônio adrenocorticotrópico em casos de neuropatias desmielinizantes aquílicas. Isto porque: 1) Dispondo de medicamento razoàvelmente ati. vo como a vitamina $B_{12}$ e sem possuirmos ainda experiência suficiente com o ACTH nesses casos, não nos pareceu lícito avaliar os resultados tardios dêsse hormônio, privando o doente de terapêutica já consagrada; por isso, nos dois primeiros casos, após o ACTH receitamos a vitamina $\mathrm{B}_{12}$; entretanto, nos dois últimos casos, em vista dos resultados relativamente satisfatórios obtidos com o ACTH, deixamos de prescrever os medicamentos clássicos, no sentido de obter, futuramente, uma avaliação dos resultados tardios. 2) Empregamos a designação "degeneração combinada da medula" por se tratar de expressão consagrada pelo uso, embora não possamos afirmar, exclusivamente com base nos dados clínicos, a existência de esclerose dorsolateral em todos os quatro casos. A propósito da casuística, cumpre ressalvar que nossos resultados, baseados em tão pequeno material, representam apenas dados preliminares para uma avaliação real da ação terapêutica do hormônio adrenocorticotrópico em tais casos.

Fazendo cuidadosa revisão da literatura não conseguimos encontrar qualquer referência ao emprêgo do ACTH ou cortisona nas degeneraçóes combinadas da medula; assim, no trabalho de Merritt ${ }^{1}$, que reviu cêrca de 200 casos de moléstias neurológicas tratados com êsses hormônios, nenhum caso de mielose funicular é referido. Quanto à anemia perniciosa, as referências são muito escassas: Gardner ${ }^{2}$ observou, em um caso, discreto aumento dos reticulócitos e da policromatofilia nos megaloblastos da medula óssea; Witts e col. ${ }^{3}$ nada referem a respeito e Heilmeyer ${ }^{4}$ apenas consigna a possibilidade de transitório estímulo da medula óssea.

Trabalho da Clínica Neurológica da Fac. Med. da Univ. de São Paulo (Serviço do Prof. Adherbal 'Tolosa), apresentado no Departamento de Neuro-Psiquiatria da Associação Paulista de Medicina em 5 março 1953.

* Assistentes. 
Fomos levados a tentar o emprêgo do ACTH nas neuraquilias em vista de que os resultados obtidos com a vitamina $B_{12}$ - que é, seguramente, o medicamento mais eficaz de que atualmente dispomos ${ }^{5}$ — não são ainda completamente satisfatórios em relação às manifestações neurológicas da moléstia, o que possivelmente se deve atribuir ao fato de que ela visa especìficamente as manifestações hematológicas. $O$ emprêgo da adrenocorticotropina se justifica, segundo cremos, se admitirmos a etiopatogenia alérgica dos processos desmielinizantes primários das neuraquilias. Hallervorden ${ }^{6}$ enquadrou as mieloses funiculares entre as moléstias desmielinizantes exógenas, inespecíficas; Ferraro $^{7}$ colocou-as entre as moléstias primitivamente desmielinizantes, admitindo, porém, a etiologia carencial como mais provável. Não cabe entrar aqui na discussão sôbre a unidade ou dualidade de fatôres (hemógeno e neurógeno) responsáveis pelo quadro clínico da moléstia de Addison-Biermer. Lembremos apenas que, para os adeptos da teoria alérgica das encefalomielites experimentais ${ }^{s}$, as proteínas alimentares deficientemente metabolizadas poderiam constituir um alérgeno capaz de provocar fenômenos desmielinizantes no sistema nervoso. Ora, em vista da aquilia existente nos casos em aprêço, é razoável admitir que, com maior razão, ocorra um distúrbio no metabolismo protêico, que conduziria à absorção de frações com poder neurossensibilizador. Por conseguinte, o uso do ACTH poderia atuar no processo de des. mielinização primária, prevenindo novas lesões, provocando a regressão das recentes; nunca, porém, como é evidente, atuaria sôbre os processos já constituídos, sejam as degeneraçóes primárias, sejam as funiculoses secundárias. Convém lembrar que o emprégo da adrenocorticotropina em casos de neuraquilia já fôra sugerido por ocasião de um simpósio sôbre neuranemias ${ }^{9}$, realizado há um ano no Departamento de Neuro-Psiquiatria da Associação Paulista de Medicina.

\section{METODO}

Foi empregado o hormônio adrenocorticotrópico (ACTH "A rmour"), na dose de $12,5 \mathrm{mg}$ diluídos em $500 \mathrm{ml}$ de soluto glicosado a $10 \%$, administrados gôta a gôta na veia, na velocidade aproximada de 20 gôtas/minuto, durante 8 horas. A todos os pacientes foi administrado cloreto de potássio, na dose de 1 a 2 diárias; dieta com restrição de sódio; cuidados habituais do tratamento com êsse hormônio. Não foi feita qualquer outra medicação associadı. Nos casos 1 e 2 fôra empregada precedentemente a vitamina $B_{12}$, que determinara discreta melhora no primeiro caso e nítida no segundo; nos casos 3 e 4 os anti-histamínicos jạ́ haviam sido usados antes do $\mathrm{AC}^{\prime} \Gamma \mathrm{H}$, tendo proporcionado, em ambos, discreta piora do quadro neurológico. Aliás, cremos que essas drogas apenas poderão atuar como preventivos do processo desmielinizante, sendo indicadas, possivelmente, em pacientes de moléstia de Addison-Biermer portadores apenas do quadro hematológico.

Procuramos avaliar quantitativamente a evolução neurológica, baseando-nos em uma tabela de Alexander 10, preconizada para o estudo da ação terapêutica na esclerose múltipla; essa tabela foi por nós adaptada à sintomatologia prevalente na mielose funicular, permitindo, assim, uma impressão mais objetiva das modificações clinicas verificadas (quadro 2). Entre os vários dados quantitativos, cabe realçar o maior rigor com que podem ser estudados os distúrbios da sensibilidade 
vibratória (através dos níveis raqueanos, pesquisados com diapasão de 256 duplas vibrações por segundo, e da determinação dos limiares, com o auxílio do "Biothesiometer"), cuja importância no quadro clínico é desnecessário encarecer 11.

\begin{tabular}{|c|c|c|c|c|c|c|c|c|c|c|c|}
\hline \multirow{2}{*}{$\begin{array}{l}\text { CLSO } \\
\text { REG.HC }\end{array}$} & \multirow{2}{*}{$\begin{array}{l}\text { SERO } \\
\text { IDADE } \\
\text { COR }\end{array}$} & \multicolumn{2}{|c|}{ SIBTOULS } & \multirow{2}{*}{$\begin{array}{c}\text { TRAF' } A \text { NTERIOR } \\
\text { RESTLTADO }\end{array}$} & \multirow{2}{*}{$\begin{array}{l}\text { DOSE } \\
\text { TOTAL } \\
(\operatorname{mg} 8)\end{array}$} & \multirow{2}{*}{ 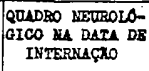 } & \multicolumn{2}{|c|}{ HEWOGRALA } & \multirow[t]{2}{*}{ roscoesure } & \multicolumn{2}{|c|}{ TATGI } \\
\hline & & Imicio & ACRAVACZO & & & & Eo & tho & & $\Delta$ & $\mathbf{F}$ \\
\hline $\begin{array}{l}\text { R.H.S.S. } \\
227034\end{array}$ & $\begin{array}{l}\text { H } \\
28 \\
B\end{array}$ & $\begin{array}{c}12 \\
\text { meses }\end{array}$ & $\begin{array}{l}1 \\
\text { mês }\end{array}$ & $\begin{array}{l}\mathrm{B}_{12}+\text { Vitar. } \\
\text { Welhora diser. }\end{array}$ & 630,0 & $\begin{array}{l}\text { Dorsal } \\
\text { Porifórico } \\
\text { Lateral }\end{array}$ & 4,3 & 12,6 & Bormobl. & 0 & - \\
\hline 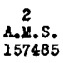 & $\begin{array}{l}M \\
43 \\
P t\end{array}$ & $\begin{array}{c}48 \\
\text { mosen }\end{array}$ & $\begin{array}{c}6 \\
\text { meses }\end{array}$ & $\begin{array}{l}\text { B12 (em 1950) } \\
\text { Hoinora acent. }\end{array}$ & 175,0 & $\begin{array}{c}\text { Dorsal } \\
\text { Per1fer1co }\end{array}$ & 0.8 & 1,9 & Vogalobl. & 0 & 20 \\
\hline $\begin{array}{c}3 \\
\text { T.L. } \\
287204\end{array}$ & $\begin{array}{c}F \\
49 \\
B\end{array}$ & $\begin{array}{c}24 \\
\text { meses }\end{array}$ & meses & $\begin{array}{l}\text { Anti-histamin. } \\
\text { Fiora discr. }\end{array}$ & 225,0 & $\begin{array}{c}\text { Dorsal } \\
\text { Poriferico }\end{array}$ & 2,4 & 7,8 & Normobl. & 0 & 20 \\
\hline $\begin{array}{c}\text { J,P.Y. } \\
276884\end{array}$ & $\begin{array}{c}M \\
46 \\
B\end{array}$ & $\begin{array}{c}14 \\
\text { moses }\end{array}$ & $-\infty$ & $\begin{array}{l}\text { Anti-histamin. } \\
\text { inal torado }\end{array}$ & 252,5 & $\begin{array}{l}\text { Dorsal } \\
\text { Porifórioo } \\
\text { Lateral }\end{array}$ & 4,2 & 11,0 & $\begin{array}{c}\text { Megalobl. } \\
\text { parcial }\end{array}$ & 0 & 16 \\
\hline
\end{tabular}

Quadro 1 - Material: Eo, eritrócitos (milhões por $\mathrm{mm}^{3}$ ) ; $\mathrm{Hb}$, hemoglobina $(\mathrm{g} / 100 \mathrm{ml})$.

\section{MATERIAL}

Nosso material consta de 4 casos* de neuropatia desmielinizante aquílica, cujos elementos fundamentais estão expostos no quadro 2. Pode-se notar que, no caso 1, não havia desordens hematológicas; no caso 2, tratava-se de uma recidiva hêmato-neurológica grave em indivíduo portador de anemia megaloblástica; no caso 3, o quadro hematológico foi considerado como correspondente a anemia verminótica (eosinofilia observada pelo exame da medula óssea), apesar do caráter hipercrômico inicial e da negatividade de repetidos exames de fezes; no caso 4, apesar da ausência de anemia evidente, foi verificada megaloblastose parcial na medula óssea. Em todos os casos havia aquilia, resistente à histamina.

As características das manifestações neurológicas podem ser apreciadas pelo exame do quadro 3. Em todos os casos estavam presentes fenômenos indicativos de lesão periférica e dos funículos dorsais da medula; em dois havia também in. dícios clínicos de lesão das vias corticospinais. - Fm todos os casos, a sintomatologia se iniciara mais de um ano antes da época em que o tratamento roi instituído; nos três primeiros, contudo, as manifestações neurológicas haviam sofrido agravação relativamente recente (de um a seis meses).

\section{RESULTADOS}

$\mathrm{Na}$ aplicação da tabela do quadro 2 a nossos casos, obtivemos os dados referidos no quadro 3 . A apreciação dêste revela, entre outros fatos, que foi em relação às síndromes funicular dorsal e piramidal que os efeitos mais se fizeram sentir; tal melhora se traduziu na recuperação da marcha em condições satisfatórias nos casos 3 e 4 , dando ensejo a que se pudesse iniciar nesses pacientes, com grande proveito, a reeducação mus: cular. E' interessante assinalar que o caso 2 , cujas melhoras foram as menos evidentes da série, era justamente aquêle em que as desordens rieu-

* O caso 4 foi incluído em nosso material após a apresentação dêste trabalho no Departamento de Neuro-Psiquiatria da Associação Paulista de Medicina. 


\begin{tabular}{|c|c|c|c|}
\hline $\operatorname{sinizj}$ & IATEISIDLDE & FUNTOS & OBSERVAÇOES \\
\hline 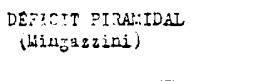 & $\begin{array}{l}\text { zueda rápla } \\
\text { zuoda lenta } \\
\text { Oscilaçós-Fadiga }\end{array}$ & $\begin{array}{r}15 \\
8 \\
2\end{array}$ & $\begin{array}{l}\text { Lado-liembro } \\
\text { Lado-Lembro } \\
\text { Lado-Membro }\end{array}$ \\
\hline 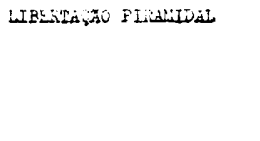 & $\begin{array}{l}\text { Eabinsici } \\
\text { Rose-Hirschberg } \\
\text { Eiperreflexia } \\
\text { Clono } \\
\text { Hipertonla ++ } \\
\text { Bipertonia * }\end{array}$ & $\begin{array}{r}10 \\
5 \\
5 \\
2 \\
5 \\
2\end{array}$ & $\begin{array}{l}\text { Lado } \\
\text { Lado } \\
\text { Lado-Reflezo" } \\
\text { Lado-Local } \\
\text { Lado-Lembro } \\
\text { Lado-Lembro }\end{array}$ \\
\hline פखण & $\begin{array}{l}\text { Só fica de pé can } \\
\text { apôio (ñ̃o anda) } \\
\text { Romberg simples } \\
\text { Konberg sensibilizado }\end{array}$ & $\begin{array}{r}35 \\
25 \\
5\end{array}$ & - \\
\hline 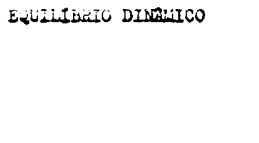 & $\begin{array}{l}\text { Acamado } \\
\text { Häo anda, mas fíca do } \\
\text { sế apôio } \\
\text { Só ando com apôio } \\
\text { :Larcha atáxica ++ } \\
\text { Larcha atáxica + }\end{array}$ & $\begin{array}{r}40 \\
20 \\
15 \\
10 \\
5\end{array}$ & $\begin{array}{l}- \\
= \\
=\end{array}$ \\
\hline COOIUERACAO AFELDICULAR & $\begin{array}{l}\text { Ataxia ++ } \\
\text { Ataxia }+\end{array}$ & $\begin{array}{l}5 \\
2\end{array}$ & $\begin{array}{l}\text { Lado-i:ambro } \\
\text { Lado-Liembro }\end{array}$ \\
\hline YLOTONLA HUSCU:AB & $\begin{array}{l}\text { Hipotonia }+ \\
\text { Hipotonia }\end{array}$ & $\begin{array}{l}5 \\
2\end{array}$ & $\begin{array}{l}\text { Lado-liembro } \\
\text { Lado-Liembro }\end{array}$ \\
\hline HIPO OU ARREFLEGLLA & $\begin{array}{l}\text { Arreflexig } \\
\text { Hiporreflexia }\end{array}$ & $\begin{array}{l}5 \\
2\end{array}$ & $\begin{array}{l}\text { Lado-Reflexo } \\
\text { Lado-Refloxo }\end{array}$ \\
\hline ARTEESTESIA & 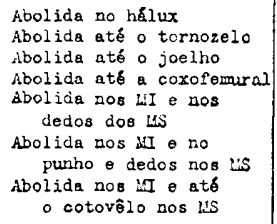 & $\begin{array}{r}5 \\
10 \\
15 \\
25 \\
30 \\
40 \\
50\end{array}$ & $\begin{array}{l}\text { Lado } \\
\text { Lado } \\
\text { Lado } \\
\text { Lado } \\
\text { Lado } \\
\text { Lado } \\
\text { Lado }\end{array}$ \\
\hline PALEZSTESIA & $\begin{array}{l}\text { Abolida no sacro } \\
\text { Abolida ate } C_{1}\end{array}$ & $\begin{array}{r}1 \\
25\end{array}$ & - \\
\hline 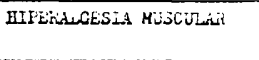 & $\begin{array}{l}\text { Hiperalgesia ++ } \\
\text { Hiporalgesia + }\end{array}$ & $\begin{array}{l}5 \\
2\end{array}$ & $\begin{array}{l}\text { Lado-Lembro } \\
\text { Lado-Lembro }\end{array}$ \\
\hline HIHCESTÉA SUY & $\begin{array}{l}\text { lio } \mathrm{p}^{8} \\
\text { lo ps e perna } \\
\text { Até } \mathrm{L} \\
\text { Até o tronco } \\
\text { dtingindo também os } Y\end{array}$ & $\begin{array}{l}2 \\
5 \\
10 \\
30 \\
50\end{array}$ & $\begin{array}{l}\text { Lado } \\
\text { Lado } \\
\text { Lado } \\
= \\
\text { - }\end{array}$ \\
\hline FAEESTESLAS & $\begin{array}{l}\text { Farestesias ++ } \\
\text { Earestesias + }\end{array}$ & $\begin{array}{l}5 \\
i\end{array}$ & $\begin{array}{l}\text { Lado-Liembro } \\
\text { Lado-Lenbro }\end{array}$ \\
\hline 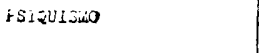 & $\begin{array}{l}\text { Distúrbios intensos } \\
\text { Distúrbios discretos }\end{array}$ & 20 & - \\
\hline
\end{tabular}

Quadro 2 - Tabela utilizada para avaliação quantitativa da sintomatologia neurológica em casos de neuropatia des. mielinizante aquílica (adaptada de uma tabela de Alexander ${ }^{10}$ ): $\quad$ Reflexos aquiliano, patelar. estilo-radial, bicipital; * clono do pé, rótula e punho.

rológicas eram mais discretas, apesar da gravidade do quadro henatológico. Importa salientar também a eosinofilia observada no caso 3, que, em vista da negatividade dos exames de fezes, talvez seja simples tradução de uma reação alérgica, o que estaria de acôrdo com o fato de que nesse caso foi obtido o maior índice de melhora com o emprêgo do ACTH.

Em conclusão, êste estudo preliminar em 4 casos de neuropatia desmielinizante aquílica permite admitir uma ação imediata favorável do ACTH; a exata avaliação do efeito dêste hormônio em tais moléstias só será possível após maior experiência, baseada em mais volumosa casuísti- 
ca e no seguimento dos pacientes, bem como no estudo comparativo dêsses efeitos com os obtidos por meio dos demais medicamentos correntemente utilizados (extrato hepático, ácido fólico e vitamina $B_{12}$ ).

\begin{tabular}{|c|c|c|c|c|c|c|c|c|}
\hline \multirow{2}{*}{$\frac{\text { SIMTOMUTOLOGIA }}{\text { MISUROLOGICA }}$} & \multicolumn{2}{|c|}{ CASO 1} & \multicolumn{2}{|c|}{ CASO 2} & \multicolumn{2}{|c|}{ CASO 8} & \multicolumn{2}{|c|}{ CASO 4} \\
\hline & ANTES & DEPOIS & ANTES & DBPOIS & ANTES & DEPOIS & ANTES & DEPOIS \\
\hline $\begin{array}{l}\text { Deficit } \\
\text { piramidal }\end{array}$ & 0 & 0 & 0 & 0 & 4 & 0 & 8 & 4 \\
\hline $\begin{array}{l}\text { Libertação } \\
\text { piranidal }\end{array}$ & 10 & 0 & 0 & 0 & 0 & 0 & 10 & 10 \\
\hline $\begin{array}{l}\text { Equilifibrio } \\
\text { eatátioo }\end{array}$ & 35 & 6 & 5 & 5 & 15 & 5 & 16 & 15 \\
\hline $\begin{array}{l}\text { Equilibrio } \\
\text { dinfmico }\end{array}$ & 6 & 5 & 6 & 5 & 15 & 5 & 15 & 10 \\
\hline $\begin{array}{l}\text { Coordenactlo } \\
\text { apond I cular }\end{array}$ & 0 & 0 & 0 & 0 & 10 & 4 & 14 & 10 \\
\hline $\begin{array}{l}\text { Hipotonia } \\
\text { misoular }\end{array}$ & 0 & 0 & 0 & 0 & 4 & 4 & 4 & 4 \\
\hline $\begin{array}{l}\text { H1po ou ar- } \\
\text { refloxia }\end{array}$ & 20 & 14 & 8 & 8 & 20 & 8 & 2 & 2 \\
\hline Artrostosia & 2 & 0 & 0 & 0 & 0 & 0 & 15 & 10 \\
\hline $\begin{array}{l}\text { Pelostesia: } \\
\text { Raque } \\
\text { Limiares }\end{array}$ & $\frac{2}{-}$ & 2 & $\begin{array}{c}6 \\
12,30\end{array}$ & $\begin{array}{l}3 \\
8,00\end{array}$ & $\begin{array}{l}18 \\
11,50\end{array}$ & $\begin{array}{l}18 \\
9,35\end{array}$ & $\begin{array}{c}5 \\
6,55\end{array}$ & $\begin{array}{l}4 \\
6,10\end{array}$ \\
\hline $\begin{array}{l}\text { Eiperalgesia } \\
\text { muscular }\end{array}$ & 4 & 4 & 4 & 4 & 0 & 0 & 10 & 4 \\
\hline $\begin{array}{l}\text { Hipoostosia } \\
\text { euperficial }\end{array}$ & 20 & 20 & 0 & 0 & 0 & 0 & 0 & 0 \\
\hline Perostesias & 8 & 4 & 0 & 0 & 20 & 20 & 10 & 4 \\
\hline CONPUTO TOTAL & 86,00 & 54,00 & 40,30 & 33,00 & 117,50 & 73,35 & 114,55 & 83,10 \\
\hline WELHORA PERCENTUAL & \multicolumn{2}{|c|}{$37,2 \%$} & \multicolumn{2}{|c|}{$28,1 \%$} & \multicolumn{2}{|c|}{$37,6 \%$} & \multicolumn{2}{|c|}{$27,5 \%$} \\
\hline COLPUTO MEDIO & \multicolumn{4}{|c|}{ Inicial } & $\begin{array}{l}89,6 \\
63,8\end{array}$ & \multicolumn{3}{|c|}{63,8} \\
\hline \multicolumn{2}{|c|}{ LBLHORA FERCENTCAL WEDIA } & & & & \multicolumn{4}{|l|}{$28,8 \%$} \\
\hline
\end{tabular}

Quadro 3 - Avaliação quantitativa dos resultados do emprêgo do ACTH em nosso material.

\section{RESUMO}

Os autores apresentam os resultados imediatos do emprêgo do ACTH em 4 casos de neuropatia desmielinizante aquílica. Justificam o uso dêsse hormônio em vista da possível patogenia alérgica dos processos desmielinizantes primários. O ACTH foi administrado por via intravenosa, sem qualquer outra medicação associada que pudesse interferir nos resultados. Êstes foram avaliados do ponto de vista quantitativo e evidenciaram discreta ação benéfica do hormônio, avaliada em $28,8 \%$, em média. A decisão quanto ao efeito do ACTH nas mieloses funiculares só será possível após maior experiência, baseada em mais volumosa casuística e no segui- 
mento dos pacientes, bem como no estudo comparativo dêsses resultados com os obtidos com o emprêgo dos demais medicamentos de uso corrente.

\author{
SUMMARY
}

\title{
ACTH in subacute combined degeneration of the spinal cord. Preliminary results.
}

Report on the immediate results of the use of ACTH in 4 cases of achylic demyelinating neuropathy. This treatment is justified by the possible allergic pathogenesis of the primary demyelinating processes. Dayly dosis of $12.5 \mathrm{mgm}$ of corticotropin were given by continuous drip method, without any other associated drug which could interfere in the results. These were estimated on a semi-quantitative basis and evidenced slight beneficial effects of the hormone, which averaged 28.8 per cent. The accurate evaluation of the effects of corticotropin in subacute combined degeneration of the cord demands larger experience, based on greater number of cases and follow up of the patients, as well as the comparative study of such effects and the results obtained with classic treatment (liver extract, folic acid and vitamin $B_{12}$ ).

\section{BIBLIOGRAFIA}

1. Merritt, H. H. - Corticotropin and cortisone in diseases of the nervous system. Yale J. Biol. a. Med., 24:466-473 (junho) 1952. 2. Gardner, F. - Discussão do trabalho: ACTH in leukemia. Blood, 5:791 (agôsto) 1950. 3. Witts, L. J., Britton, L. J. C., Davidson, L. S. P., Davis, L. J., Haddow, A., Whitby, S., Wilkinson, J. F. e Mollison, P. L. - The treatment of blood disorders with ACTH and cortisone. Brit. M. J., 1:1261-1262 (14 junho) 1952. 4. Heilmeyer, L. - AC'IH und Cortisontherapie bei nicht leukhämischen Bluterkrankungen. Acta Haematol., 7:206-216 (abril) 1952. 5. Canelas, H. M. e Jamra, M. A. -Estudo comparativo dos efeitos hematológicos e neurológicos do extrato hepático, ácido fólico e vitamina $B_{12}$ no tratamento da anemia perniciosa de Addison-Biermer. A rq. Neuro-Psiquiat,, 11:229-246 (setembro) 1953, 6. Hallervorden, J. Die zentralen Entmarkungskrankheiten. Deutsche '/tschr. f. Nervenh., 150:201239, 1940. 7. Ferraro, A. - Primary demyelinating processes of the central nervous system. An attempt at unification and classification. Arch. Neurol. a. Psychiat., 37:1100-1160 (maio) 1937. 8. Ferraro, A., Roizin, I. e Cazzullo, C. L. - Experimental studies in allergic encephalomyelitis. Prevention and production. Note III. J. Neuropathol. a. Exper. Neurol., 9:18-28 (janeiro) 1950. 9. Canelas, H. M. - Diagnóstico e tratamento das neuranemias. Rev. Paulista de Med., 41:255-256 (outubro) 1952. Resumo. 10. Alexander, L. - New concept of critical steps in course of chronic debilitating neurologic disease in evaluation of therapeutic response. A rch. Neurol. a. P'sychiat., 66:253-271 (setembro) 1951. 11. () Jamra, M. A., Canelas, H. M. e Bittencourt, J. M. 'I. - O ácido fólico no tratamento da síndrome nervosa da anemia perniciosa. Fistudo do comportamento da sensibilidade vibratória. Arq. Neuro-Psiquiat., 6:301-333 (dezembro) 1948; b) Canelas, H. M. - Distúrbios neurológicos nos estados carenciais aquílicos. Arq. Neuro-Psiquiat., 10:1-40 (março) 1952. 